\title{
Previsão da exportação brasileira de açúcar avaliada por meio de gráficos de controle de resíduos
}

\author{
Brazilian sugar export forecast by means of residual control charts
}

\author{
Valentina Wolff Lirio', Renan Mitsuo Ueda", Bianca Reichert"', \\ Adriano Mendonça Souzalv
}

\begin{abstract}
RESUMO
A produção e a exportação do açúcar são fatores importantes para o avanço da economia brasileira, uma vez que o Brasil é o maior produtor de açúcar e responsável por quase metade das exportações mundiais da commodity. O objetivo da pesquisa é monitorar a quantidade de açúcar exportada no período de janeiro de 2000 a abril de 2019, a partir de gráficos de controle de resíduos com prétratamento por meio da modelagem Autorregressiva Integrada de Médias Móveis (ARIMA). Os dados utilizados no estudo foram coletados do site Portal Única. Optou-se pela aplicação da modelagem ARIMA, pois os dados apresentaram evidências de não estacionariedade e valores autocorrelacionados. $O$ melhor modelo para prever a exportação brasileira de açúcar foi o $\operatorname{SARIMA}(1,1,1)(1,0,1)_{6}$, devido ao comportamento sazonal da série que pode estar relacionado ao período de plantio/colheita da cana-deaçúcar. Ao elaborar o gráfico de controle residual, foi possível observar a presença de outliers no limite superior nos meses de outubro de 2012 e fevereiro de 2016, os quais caracterizaram uma exportação real do açúcar muito maior que exportação prevista.
\end{abstract}

Palavras-chave: Modelos ARIMA; Gráficos de Controle; Exportação do Açúcar.

\begin{abstract}
Sugar production and exportation are important factors for the Brazilian economy, because Brazil produces the largest amount of sugar and accounts for almost half of the world's sugar exports. This research aimed to monitor the sugar export from January 2000 to April 2019, by means of residual control charts with pretreatment of autoregressive integrated moving average (ARIMA) models. The data used in the study were collected from the Portal Única website. We opted for the application of ARIMA modeling because the data was not stationarity and presented autocorrelated values. The best model to predict the Brazilian sugar exports was SARIMA $(1,1,1)(1,0,1)_{6}$ due to the seasonal behavior of the series, which may be related to the sugarcane planting and harvesting period. It was possible to observe the presence of upper-limit outliers in the residual control chart, in October 2012 and February 2016, which characterize a sugar exports higher than forecasted exports.
\end{abstract}

Keywords: ARIMA models; Control Charts; Sugar Export. 


\section{INTRODUÇÃO}

Nas últimas décadas, o setor sucroenergético brasileiro sofreu uma revolução tecnológica significativa. Atualmente, o país é considerado o maior produtor global de açúcar, responsável por $20 \%$ da produção global, $45 \%$ da exportação mundial e as plantações de cana-de-açúcar corresponde a 1,2\% de todo o território brasileiro (ÚNICA, 2019).

A partir de 2003, aconteceu uma aceleração dos investimentos em novas usinas motivada pelo crescimento da demanda de açúcar no mercado internacional. Após a crise financeira de 2008 , os investimentos no setor cessaram e a expansão dos canaviais foi comprometida, já que grande parte das empresas se encontravam altamente endividadas, esse cenário foi potencializado pelo aumento da oferta mundial de açúcar. As quedas de produtividade, causadas pela redução nos tratos com os canaviais, a idade avançada da lavoura, a mecanização e os problemas climáticos, começaram a ser revertidas na safra 2013/2014 (NOVACANA, 2014).

No âmbito nacional, os maiores produtores de açúcar são: o estado de São Paulo, que produz em torno de $60 \%$ de toda a cana, açúcar e etanol do país, e o segundo maior produtor é o estado do Paraná, com 8\% da cana moída no Brasil (NOVACANA, 2008). A pergunta de pesquisa a ser respondida ao longo do artigo, pode ser relatada da seguinte maneira: como se dá o monitoramento da quantidade de açúcar exportada no período de janeiro de 2000 a abril de 2019?

Esta pesquisa investiga a exportação brasileira de açúcar, por meio de previsões, a fim de apoiar decisões estratégicas do setor. O objetivo da pesquisa é monitorar a quantidade de açúcar exportada no período de janeiro de 2000 a abril de 2019, a partir de gráficos de controle de resíduos com pré-tratamento por meio da modelagem Autorregressiva Integrada de Médias Móveis (ARIMA).

A proposta do artigo é relevante, dado que a partir dele se poderá compreender o comportamento da exportação brasileira de açúcar, por meio da análise de séries temporais, e com isso fornecer subsídios para tomada de decisões estratégicas no setor. Além disso, conhecer e prever o comportamento da exportação brasileira do 
açúcar é uma informação relevante para a balança comercial do país, já que o açúcar é um dos principais produtos exportados.

O presente trabalho encontra-se estruturado em cinco seções, que pode ser sumarizadas da seguinte forma: a primeira refere-se a parte introdutória; a segunda traz o referencial teórico; a terceira trata dos métodos e dados que darão suporte à aplicação empírica; a quarta refere-se a análise e discussão dos resultados e a quinta diz respeito as considerações finais.

\section{REFERENCIAL TEÓRICO}

Nesta seção é apresentado o referencial teórico sobre a temática que envolve o trabalho: ciclo do açúcar no Brasil (2.1); produção e exportação de açúcar em âmbito nacional (2.2); modelo autorregressivo integrado e de médias móveis (ARIMA)(2.3); gráficos de controle (2.4).

\subsection{Ciclo do Açúcar no Brasil}

Segundo Ferlini (2010), entre meados do século XVI e XVIII, ocorreu o ciclo do açúcar durante o período da história compreendido como Brasil Colonial. Neste período, a produção de açúcar foi a principal atividade econômica, voltada para a exportação e desenvolvida nos engenhos do Nordeste brasileiro.

Inicialmente, Portugal tinha muita preocupação em relação ao modo como iria explorar o Brasil, dado que era urgente a necessidade de tornar a colônia lucrativa. Assim, o rei Dom João III enviou Martim Afonso de Souza à América, em 1533.0 objetivo era que Martim Afonso explorasse o território brasileiro, numa forma de proteger a colônia de invasões estrangeiras. Consigo o explorador português trouxe mudas de cana-de-açúcar. Martim Afonso de Souza fundou a Vila de São Vicente (no litoral do atual estado de São Paulo), onde instalou o primeiro engenho de açúcar no Brasil. (FABER, 2016). A economia do açúcar foi responsável pela consolidação da colonização, por meio da ocupação de parte da costa brasileira, sendo utilizada mão de obra de escravos africanos. Porém, a economia açucareira começou a entrar em crise na segunda metade do século XVII, com a expulsão dos holandeses do nordeste brasileiro. Empreendedores holandeses foram para a região das Antilhas produzir açúcar. Logo, 
os holandeses se tornaram um forte concorrente, uma vez que vendiam o açúcar mais barato na Europa, além de controlarem o transporte e comércio do produto. Desta maneira, os holandeses conquistaram o mercado consumidor europeu, iniciando uma forte crise na economia açucareira no Brasil. Essa crise, acentuou-se ainda mais em meados do século XVIII, período em que a economia brasileira passou a se voltar para o ouro da região das Minas Gerais. A região Sudeste passou a atrair investimentos, a capital foi transferida de Salvador para o Rio de Janeiro e o Ciclo do Açúcar chegou ao fim (FERLINI, 2010).

\subsection{Produção e exportação de açúcar em âmbito nacional}

Historicamente, o Brasil é o maior produtor de açúcar do mundo. O açúcar brasileiro chega a todos os cantos do globo e ajuda a combater a desnutrição, sendo uma importante fonte natural e acessível de energia para os indivíduos. É um ingrediente fundamental no preparo de alimentos para obtenção de sabor e consistência e faz parte da cultura e do dia-a-dia dos brasileiros (ÚNICA, 2019).

A partir de 2003, ocorreu uma aceleração nos investimentos em usinas que foi motivado pelo crescimento da demanda de açúcar no mercado internacional, especialmente após a reforma da política europeia para o produto e também pelo uso crescente do etanol, a partir do desenvolvimento dos veículos com motores Flex Fuel no país. Com isso, a produção de cana-de-açúcar passou por um significativo incremento, fundamentalmente no período compreendido entre os ciclos de 2001/2002 e 2008/2009, ano da crise econômica mundial. Nesse intervalo, a safra cresceu a um ritmo de 10,6\% ao ano, atingindo 573 milhões de toneladas. A partir de 2009/2010, até a safra 2012/2013, houve não só uma ruptura com o ritmo apresentado até então, mas a produção passou a variar negativamente em $1 \%$ ao ano (NOVACANA, 2014).

A maior parte da produção do açúcar concentra-se, principalmente, no sudeste do país. Nessa região, destaca-se o estado de São Paulo, no qual em 2009, foi responsável por cerca de $60 \%$ da produção nacional e que segue nos dias atuais como maior produtor. Todavia, novos cultivos têm se expandido principalmente na região Centro-Oeste, devido essencialmente aos menores preços de terras. Em 2009, a área 
plantada de cana era de aproximadamente 8,5 milhões de hectares, representando menos de 3\% do total de terras cultiváveis no Brasil. A cana-de-açúcar cresce melhor em regiões quentes e úmidas, com temperaturas entre 30 e 34 graus Celsius, além de que a adubação representa sem dúvidas um dos maiores custos na produção da cana (AGRIC, 2009).

A produção brasileira de açúcar passou de 16,3 milhões de toneladas em 2000, para 38,2 milhões de toneladas em 2013, ficando à frente de países como Índia e China. Diante deste cenário, o Brasil tornou-se o maior fornecedor do produto no mercado internacional, chegando a responder por metade das exportações mundiais no período ( $50 \%$ do volume total), seguido pela Tailândia que, devido a um surpreendente ritmo de crescimento de $14 \%$ ao ano na última década, já representa $14 \%$ das exportações globais (NOVACANA, 2014).

Entretanto, o Brasil deve perder para a Índia a liderança na produção de açúcar no ano-safra internacional 2018/19, pela primeira vez em décadas. Isso salienta um movimento em curso na indústria brasileira de direcionar cada vez mais cana para a produção de etanol, em uma conjuntura de baixos investimentos nas lavouras que têm resultado em produtividades cada vez menores. A mudança na liderança pela produção de açúcar deverá ter reflexo nos fluxos comerciais da mercadoria ao redor do mundo, com as exportações brasileiras perdendo fatia, enquanto a Ásia ganha espaço no mercado global e concentra estoques da commodity, especialmente na Índia (REUTERS, 2018).

A Índia tem grandes condições de intensificar a exportação do açúcar e o Brasil tende a recuar as exportações no ano-safra (2018/19), em torno de 9 milhões de toneladas. A condição de maior produtor não tem previsão para ser recuperada, dado que o preço do açúcar está baixo e do etanol e gasolina estão mais altos. O Brasil vinha liderando o ranking de produção global desde que a União Europeia perdeu essa posição na década de 1990 (BORGES, 2018).

A perda de liderança na produção global de açúcar pelo Brasil é relativizada por alguns especialistas, que ainda veem o país como principal influência para as cotações da commodity na Bolsa de Nova York (ICE Futures US), além de que, é cedo para dizer 
se a Índia vai repetir em 2019/20 a grande safra de 2018, até porque as usinas estão enfrentando dificuldades para pagar fornecedores de cana. Segundo Plinio Nastari, presidente da Datagro, a redução de produção de açúcar no Brasil denota um enorme protagonismo, que ajuda a mitigar essa pressão sobre as cotações e faz com que o Brasil siga sendo um leme muito importante para o mercado (REUTERS, 2018).

\subsection{Modelo Autorregressivo Integrado e de Médias Móveis (ARIMA)}

A metodologia desenvolvida por Box e Jenkins (1970) baseia-se na ideia de que as observações possuem uma ligação, a qual influencia o comportamento atual e futuro da série e, assim, se pode predizer os valores futuros. Esse método, também conhecido como modelo Autorregressivo Integrado de Médias Móveis (ARIMA) e possui como pressuposto básico a estacionariedade da série temporal (SOUZA, 2016). Onde o filtro AR representa a parte autorregressiva, o filtro I representa integração, a qual é utilizada para a série se tornar estacionária e MA é o filtro de médias móveis.

Para verificar a estacionariedade, utiliza-se o gráfico da série em nível a fim de identificar ciclos, tendências e comportamento da média. Entretanto, a confirmação da estacionariedade é mais precisa ao utilizar os testes de raízes unitárias. Os testes de raízes unitárias mais utilizados são: Augmented Dickey e Fuller - ADF (DICKEY; FULLER, 1981) e Kwiatkowski, Phillips, Schmidt e Shin - KPSS (KWIATKOWSKI; PHILLIPS; SCHMIDT; SHIN, 1992). Se a série não for estacionária (apresenta tendência estocástica ou sazonalidade), a mesma deverá ser diferenciada até alcançar a sua estabilidade (SOUZA, 2016). A correlação entre as observações pode ser verificada por meio das funções de autocorrelação (FAC) e de autocorrelação parcial (FACP) amostrais. Os gráficos da FAC e FACP amostrais também auxiliam na identificação do melhor filtro (AR, MA, ARMA, ARIMA ou SARIMA) e na indicação da ordem do modelo a ser utilizado na etapa de estimação (BOX; JENKINS; REINSEL, 1994).

A equação do modelo geral ARIMA ( $p, d, q)$ é dada pela Equação (1). $\phi(B) \Delta^{d} X_{t}=\theta(B) \varepsilon_{t}$.

Onde $\Delta$ representa a ordem de diferenças $d$ que a série original deve ter até atingir a estacionariedade, $X_{t}$ representa a série temporal em análise, t corresponde ao 
instante de tempo atual, $B$ é o operador de retardo, $d$ caracteriza a ordem de integração, $\phi(B)$ é o polinômio que representa a parte autorregressiva do modelo (p), $\theta(B)$ é o polinômio que representa a parte de médias móveis (q) e $\varepsilon_{t}$ é o processo ruído branco. $O$ resíduo $\widehat{\varepsilon_{t}}$ corresponde à diferença entre os valores da série original e os valores previstos pelo modelo ajustado. Esses resíduos devem ser do tipo Ruído Branco, com média zero, variância constante e valores não autocorrelacionados (MORETTIN, 2016).

Após a etapa de estimação dos modelos ARIMA, o melhor ajuste será escolhido com base no critério de seleção de modelos Akaike (AIC) e no critério de informação Bayesiano (BIC). Serão selecionados os modelos que apresentarem valores mínimos para essas estatísticas (BOZDOGAN, 1987). Estes critérios são também denominados penalizadores, pois ambos consideram o número de parâmetros dos modelos e a variância dos erros (AKAIKE, 1974; SCHWARZ, 1978).

Os critérios AIC e BIC são calculados conforme as Equações (2) e (3).

$$
\begin{aligned}
& \operatorname{AIC}(p, q)=\ln \sigma_{p, q}^{2}+\frac{2(p+q)}{N} . \\
& B I C(p, q)=\ln \sigma_{p, q}^{2}+(p+q) \frac{\ln N}{N} .
\end{aligned}
$$

Onde $p$ caracteriza o número de parâmetros autorregressivos do modelo, $q$ é o número de parâmetros de médias móveis, $N$ é o número de observações, $\sigma_{p, q}^{2}$ corresponde à variância estimada dos erros e In o logaritmo neperiano. Com o objetivo de verificar a acurácia de previsão do modelo, aplicam-se as estatísticas de previsão estimadas com os erros da modelagem. As estatísticas que serão utilizadas nesta pesquisa são: Mean Absolute Error (MAE), Mean Absolute Percentage Error (MAPE) e o coeficiente U-Theil. Tais medidas de acurácia são calculadas conforme as Equações 4, 5 e 6 .

$$
\begin{aligned}
& M A E=\frac{\sum_{t=1}^{N}\left|X_{t}\right|}{N} . \\
& M A P E=\frac{1}{N} \sum\left|\frac{X_{t}-\hat{X}_{t}}{X_{t}}\right| * 100 . \\
& U-\text { Theil }=\frac{\sqrt{\sum_{t=1}^{N}\left(X_{t}-\widehat{X}_{t}\right)^{2}}}{\sqrt{\sum_{t=1}^{N}\left(X_{t}-X_{t-1}\right)^{2}}} .
\end{aligned}
$$


Onde $X_{t}$ corresponde ao valor da série original no período $t, \hat{X}_{t}$ caracteriza o valor ajustado pelo modelo no período $t, N$ é o número de observações. O coeficiente U-Theil corresponde a comparação realizada entre os valores do modelo que foi ajustado e a previsão dita ingênua. Nesta medida de acurácia se o valor de U-Theil for maior ou igual a 1, tem-se que o erro médio do modelo ajustado é superior ou igual ao da previsão ingênua. Caso o valor de U-Theil seja menor que 1, então pode-se dizer que o modelo ajustado é melhor previsor quando comparado com a previsão ingênua.

\subsection{Gráficos de Controle}

Os gráficos de controle distinguem a variabilidade aleatória da não-aleatória. A base do gráfico de controle é a distribuição amostral, que tende a ter a curva de probabilidades associada a uma distribuição gaussiana. O gráfico de controle apresenta dois limites - calculados a partir dos dados amostrais - que separam a variação aleatória da variação não-aleatória. O valor maior corresponde ao limite superior de controle (LSC) e o valor menor é chamado de limite inferior de controle (LIC). Uma estatística amostral localizada entre esses dois limites sugere a aleatoriedade da distribuição, enquanto um valor exterior a um dos dois limites sugere a não-aleatoriedade. Nos gráficos de controle é comum a utilização do LSC três sigmas acima da linha média $(\mu+3 \sigma)$ e do LIC três sigmas abaixo desta $(\mu-3 \sigma)$ (REBELATO et al.,2006).

Para a utilização dos gráficos de controle (GC), alguns pressupostos devem ser considerados, tais como independência e não autocorrelação. Também é necessário que as observações sejam normalmente distribuídas. Se a autocorrelação estiver presente, a aplicação de gráficos de controle torna-se inadequada, pois viola os pressupostos citados acima e assim é preciso tratar a autocorrelação por meio da modelagem ARIMA (Auto Regressive Integrate Moving Average) e/ou SARIMA (Sesonal Auto Regressive Integrate Moving Average), a qual produzirá resíduos livres de autocorrelação (SOUZA et al., 2012). 


\section{MÉTODOS E DADOS}

A pesquisa teve como foco a quantidade de açúcar (ton.) exportada pelo Brasil, com dados oriundos do site Portal Única (www.unica.com.br). Os valores mensais da exportação são referentes ao período de janeiro de 2000 a abril de 2019, totalizando 232 observações.

A metodologia de Box e Jenkins (1970) foi utilizada para modelar e prever a exportação brasileira de açúcar. As etapas aplicadas da modelagem ARIMA foram:

- Identificação: o pressuposto de estacionariedade foi verificado por meio de inspeção gráfica e confirmado pelos testes de raízes unitárias (ADF e KPSS).

- Estimação: o filtro do modelo (AR, MA, ARIMA ou SARIMA) e o número de defasagens dos parâmetros foram determinados por meio dos gráficos da FAC e FACP amostrais.

- Validação: a condição de ruído branco foi verificada por meio da FAC e FACP dos resíduos e pelo teste de Ljung-Box, além dos valores dos critérios de seleção de modelos AIC e BIC.

Após selecionar o melhor modelo para prever a exportação do açúcar brasileiro, os resíduos da modelagem ARIMA foram utilizados para elaborar gráficos de controle, pois os valores originais da série são autocorrelacionados e violam o pressuposto básico de aplicação dos gráficos desenvolvidos por Walter A. Shewhart (MONTGOMERY, 1997). O gráfico de controle de medidas individuais foi elaborado com o intuito de avaliar o desempenho das previsões e verificar se o processo se encontra sob controle estatístico. Ao interpretar o gráfico, busca-se identificar a presença de outliers no limite superior ou inferior. A presença de outlier no limite superior caracterizará uma quantidade real de exportação muito maior que a quantidade prevista. Por outro lado, o outlier no limite inferior representará uma quantidade real de exportação muito menor que a quantidade prevista. Os gráficos de controle foram elaborados com o auxílio do software Statistica e o software Eviews 9 S.V. foi empregado para realizar a análise e modelagem da série, juntamente com software $R$, o qual foi utilizado para gerar o gráfico da série original com a curva de tendência. 


\section{ANÁLISE E DISCUSSÃO DOS DADOS}

Verificou-se por meio da inspeção visual da série original (Figura 1) que a mesma possui um comportamento de sazonalidade e tendência ao longo do tempo. A curva azul encontrada no gráfico representa a tendência da série, a qual foi ajustada sob os valores observados da série temporal. Assim, ajustou-se uma tendência polinomial de grau 6 para chegar próximo de explicar a tendência da série.

Figura 1 - Exportação brasileira de açúcar no período de janeiro de 2000 a abril de 2019

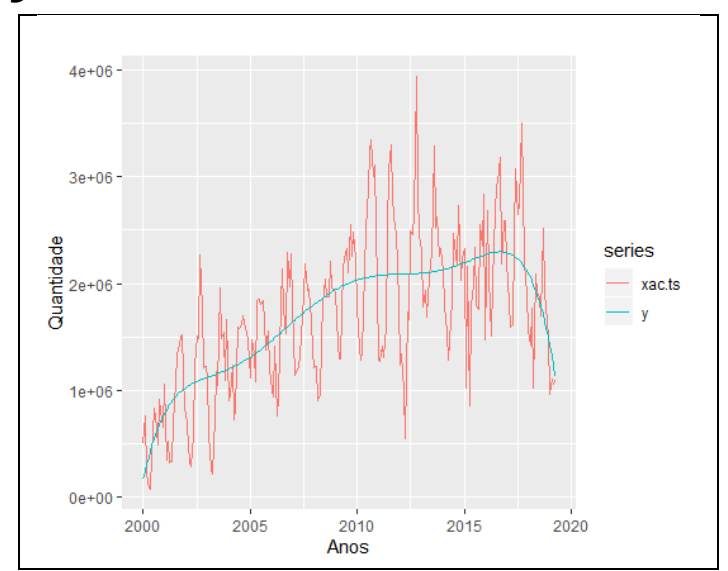

Fonte: Autores.

A fim de analisar a estacionariedade da série de exportação brasileira de açúcar, realizou-se os testes de raízes unitárias: Augmented Dickey e Fuller (ADF) e Kwiatkowski, Phillips, Schmidt e Shin (KPSS). Os resultados dos testes ADF, e KPSS estão contidos na Tabela 1 a seguir.

Tabela 1 - Teste de raízes unitárias

\begin{tabular}{|c|c|c|}
\hline Testes & Série original & Série após aplicar a $1^{\text {as }}$ diferença \\
\hline ADFa & $-0,8326(p=0,9601)^{c}$ & $-11,9870(p>0,00)^{c}$ \\
\hline KPSS $^{b}$ & $0,3188^{\mathrm{e}}$ & $0,0384^{e}$ \\
\hline
\end{tabular}

Conforme os testes ADF e KPSS, a série de exportação brasileira de açúcar se torna estacionária após a aplicação de uma diferença. Sendo assim, na Figura 2 é apresentada a série diferenciada $(\mathrm{d}=1)$ e em nível (série temporal original), representadas pelas cores vermelho e azul, respectivamente. A estacionariedade da série garantirá a estabilidade dos parâmetros a serem estimados. 
Figura 2 - Série original e diferenciada da Exportação brasileira de açúcar no período de janeiro de 2000 a abril de 2019

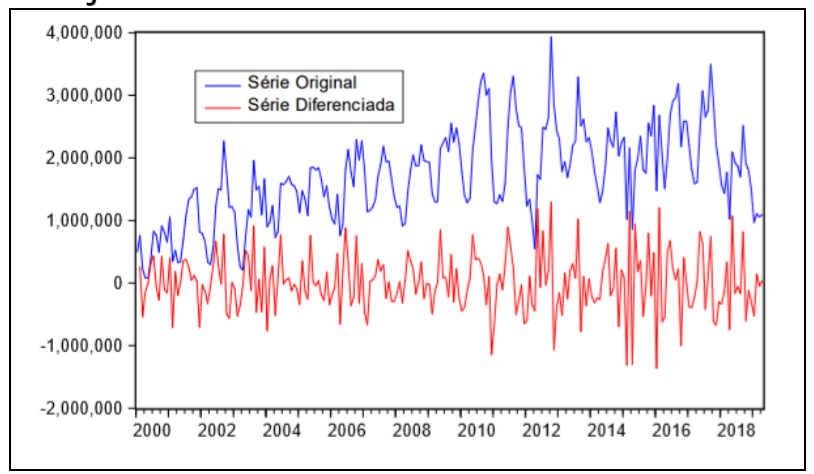

Fonte: Autores.

Na Figura 3 são apresentados os gráficos da função de autocorrelação (FAC) e da autocorrelação parcial (FACP) amostrais, com o intuito de identificar os possíveis modelos concorrentes, isto é, modelos capazes de representar o comportamento da série original da forma mais precisa.

Figura 3 - Função de autocorrelação (FAC) e função de autocorrelação parcial (FACP) amostrais

\begin{tabular}{|c|c|c|c|c|c|c|}
\hline Autocorrelation & Partial Correlation & & $A C$ & PAC & Q-Stat & Prob \\
\hline E & 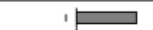 & 1 & 0.791 & 0.791 & 147.15 & 0.000 \\
\hline בע & 1' & 2 & 0.662 & 0.097 & $\begin{array}{l}250.73 \\
300.59\end{array}$ & 0.000 \\
\hline ص & : & 4 & $\begin{array}{l}0.494 \\
0.318\end{array}$ & $\begin{array}{l}-0.151 \\
-0.160\end{array}$ & $\begin{array}{l}308.59 \\
33273\end{array}$ & 0.000 \\
\hline 尚 & 4 & $\begin{array}{l}4 \\
5\end{array}$ & $\begin{array}{l}0.318 \\
0.192\end{array}$ & $\begin{array}{l}-0.760 \\
-0.001\end{array}$ & $\begin{array}{l}332.13 \\
341.55\end{array}$ & 0.000 \\
\hline ip & ia & 6 & 0.123 & 0.102 & 345.21 & 0.000 \\
\hline 巴象 r r & '包 & & 0.156 & 0.253 & 351.08 & 0.000 \\
\hline '曰 & 邑 & & 0.247 & 0.240 & 365.84 & 0.000 \\
\hline ק": & : & 9 & 0.401 & 0.287 & 405.00 & 0.000 \\
\hline 业 & קُ" & $\begin{array}{l}10 \\
11\end{array}$ & $\begin{array}{l}0.528 \\
0.655\end{array}$ & $\begin{array}{l}0.131 \\
0.186\end{array}$ & $\begin{array}{l}473.05 \\
578.40\end{array}$ & $\begin{array}{l}0.000 \\
0.000\end{array}$ \\
\hline & $\sqrt{1}$ & 12 & 0.683 & -0.001 & 693.37 & 0.000 \\
\hline ש & ' & 13 & 0.614 & -0.141 & 786.97 & 0.000 \\
\hline שـ & 1 & 14 & 0.503 & -0.092 & 850.08 & 0.000 \\
\hline ص & , & 15 & 0.346 & -0.074 & 880.12 & 0.000 \\
\hline 尚 & (4) & 16 & 0.183 & -0.095 & 888.51 & 0.000 \\
\hline i & , & 17 & 0.074 & 0.013 & 889.90 & 0.000 \\
\hline ifi, & 16 & 18 & 0.037 & 0.056 & 890.25 & 0.000 \\
\hline & 11 & 19 & 0.062 & 0.025 & 891.22 & 0.000 \\
\hline a & ini & 20 & 0.145 & -0.025 & $\begin{array}{l}896.62 \\
896.62\end{array}$ & 0.000 \\
\hline ص & $1 / 11$ & 21 & 0.281 & 0.048 & 916.93 & 0.000 \\
\hline 巨 & if & & 0.398 & -0.019 & 957.94 & 0.000 \\
\hline & i & 23 & 0.514 & 0.095 & 1026.4 & 0.000 \\
\hline 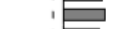 & i & 24 & 0.547 & 0.054 & 1104.6 & 0.000 \\
\hline 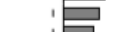 & {$[1$} & 25 & 0.479 & -0.098 & 1164.9 & 0.000 \\
\hline 与 & 11 & 26 & 0.387 & -0.012 & 1204.5 & 0.000 \\
\hline 曰 & 1) & 27 & 0.257 & 0.031 & 1221.9 & 0.000 \\
\hline & $1 \%$ & 28 & 0.115 & $\begin{array}{l}-0.032 \\
\end{array}$ & $\begin{array}{l}1225.4 \\
1206\end{array}$ & 0.000 \\
\hline 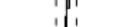 & ip & $\begin{array}{l}29 \\
30\end{array}$ & $\begin{array}{r}0.046 \\
-0.000\end{array}$ & $\begin{array}{r}0.107 \\
-0.017\end{array}$ & $\begin{array}{l}1226.0 \\
12260\end{array}$ & $\begin{array}{l}0.000 \\
0.000\end{array}$ \\
\hline 1 & $i_{11}$ & 31 & 0.038 & 0.043 & 1226.3 & 0.000 \\
\hline$\mu$ & 14. & 32 & 0.108 & -0.074 & 1229.5 & 0.000 \\
\hline 曰 & 11 & 33 & 0.231 & 0.029 & 1244.0 & 0.000 \\
\hline 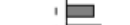 & 19 & 34 & 0.360 & 0.076 & 1279.5 & 0.000 \\
\hline & 11 & 35 & 0.446 & 0.025 & 1334.3 & 0.000 \\
\hline$\square$ & 111 & 36 & 0.473 & 0.010 & 1396.2 & 0.000 \\
\hline
\end{tabular}

Fonte: Autores.

Pela Figura 3 é possível a visualização dos componentes autorregressivos, onde o decaimento da função de autocorrelação possui a forma ondular, o que indica a presença de sazonalidade na série. Posteriormente, os parâmetros dos modelos concorrentes foram ajustados a fim de encontrar o melhor modelo de previsão (Tabela 2). Na Tabela 2 estão contemplados os modelos concorrentes, seus parâmetros e nível de significância, os valores dos critérios de seleção de modelos AIC e BIC, informação sobre a presença de ruído branco e os resultados do teste de Ljung-Box. 
Tabela 2 - Modelos ajustados a série temporal em estudo - ARIMA

\begin{tabular}{|c|c|c|c|c|c|}
\hline \multicolumn{6}{|c|}{ ARIMA $(1,1,0)$} \\
\hline Parâmetro & $p$-value & AIC & $\mathrm{BIC}$ & Ruído branco & Teste Ljung-Box \\
\hline$\varphi_{1}=-0,1919$ & 0,0007 & 28,9239 & 28,9537 & Sim & $\begin{array}{l}\text { Os resíduos não são } \\
\text { autocorrelacionados }\end{array}$ \\
\hline \multicolumn{6}{|c|}{$\operatorname{ARIMA}(1,1,1)$} \\
\hline Parâmetro & $p$-value & AIC & $\mathrm{BIC}$ & Ruído branco & Teste Ljung-Box \\
\hline $\begin{array}{l}\varphi_{1}=0,6651 \\
\theta_{1}=-0,9527\end{array}$ & $\begin{array}{l}\mathrm{p}<0,0000 \\
\mathrm{p}<0,0000\end{array}$ & 28,8364 & 28,8811 & Sim & $\begin{array}{l}\text { Os resíduos não são } \\
\text { autocorrelacionados }\end{array}$ \\
\hline
\end{tabular}

Fonte: Autores.

Dentro da família dos modelos lineares, os modelos SARIMA são utilizados para captarem os efeitos sazonais, desta forma o modelo fica especificado como SARIMA $(p, d, q)(P, D, Q) s$, se os efeitos sazonais estiverem presentes e os valores dos parâmetros representados por $\mathrm{P}, \mathrm{D}$ e Q devem ser estimados. O valor de $\mathrm{s}$ corresponderá ao tamanho da sazonalidade. Se a variável não apresentar componentes sazonais, obtémse o modelo ARIMA(p,d,q). Na Tabela 3 estão contemplados os modelos concorrentes dos modelos SARIMA, seus parâmetros, nível de significância, os valores dos critérios de seleção de modelos AIC e BIC, informação sobre a presença de ruído branco e os resultados do teste de Ljung-Box.

Tabela 3 - Modelos ajustados a série temporal em estudo - SARIMA

\begin{tabular}{|c|c|c|c|c|c|}
\hline \multicolumn{6}{|c|}{ SARIMA $(1,1,0)(1,0,0)_{6}$} \\
\hline Parâmetro & $p$-value & AIC & $\mathrm{BIC}$ & Ruído branco & Teste Ljung-Box \\
\hline$\varphi_{1}=-0,3316$ & $p<0,0000$ & & & & Os resíduos não são \\
\hline$\Phi_{6}=-0,3630$ & $p<0,0000$ & 28,8114 & 28,8561 & sim & autocorrelacionados \\
\hline \multicolumn{6}{|c|}{ SARIMA $(1,1,1)(1,0,0)_{6}$} \\
\hline Parâmetro & $p$-value & $\mathrm{AIC}$ & $\mathrm{BIC}$ & Ruído branco & Teste Ljung-Box \\
\hline$\varphi_{1}=0,4547$ & $p<0,0000$ & & & & Os resíduos não são \\
\hline$\theta_{1}=-0,8701$ & $p<0,0000$ & 28,7490 & 28,8086 & Sim & autocorrelacionados \\
\hline$\Phi_{6}=-0,3821$ & $p<0,0000$ & & & & \\
\hline \multicolumn{6}{|c|}{ SARIMA $(1,1,1)(1,0,1)_{6}$} \\
\hline Parâmetro & $p$-value & AIC & $\mathrm{BIC}$ & Ruído branco & Teste Ljung-Box \\
\hline$\varphi_{1}=0,3111$ & 0,0001 & & & & Os resíduos não são \\
\hline$\theta_{1}=-0,8549$ & $p<0,0000$ & 285415 & 286160 & Sim & autocorrelacionados \\
\hline$\Phi_{6}=-0,9997$ & $p<0,0000$ & 28,5415 & 28,6160 & sim & \\
\hline$\Theta_{1}=0,9796$ & $p<0,0000$ & & & & \\
\hline
\end{tabular}

O modelo com o menor valor para os critérios penalizadores foi o SARIMA $(1,1,1)(1,0,1)_{6}$, sendo este o modelo selecionado. Este modelo, apresenta os menores valores para os critérios AIC e BIC e parâmetros significativos ( $p$-value<0,05). Sendo assim, o melhor modelo que se ajustou aos dados da exportação brasileira de 
açúcar aponta que a série possui um autorregressivo de ordem 1 (com fator de influência positivo 0,3111), ou seja, o volume exportado de açúcar do mês anterior influencia no mês atual. Ainda, capturou um filtro de médias móveis com parâmetro negativo $(-0,8549)$, um filtro sazonal de 6 meses $(-0,9997)$ e um filtro de médias móveis sazonal (0,9796). De acordo com a FAC e a FACP amostrais não há a presença de autocorrelação dos resíduos oriundos da modelagem (Figura 4).

Figura 4 - Função de autocorrelação (FAC) e função de autocorrelação parcial (FACP) amostrais dos resíduos do modelo SARIMA $(1,1,1)(1,0,1) 6$

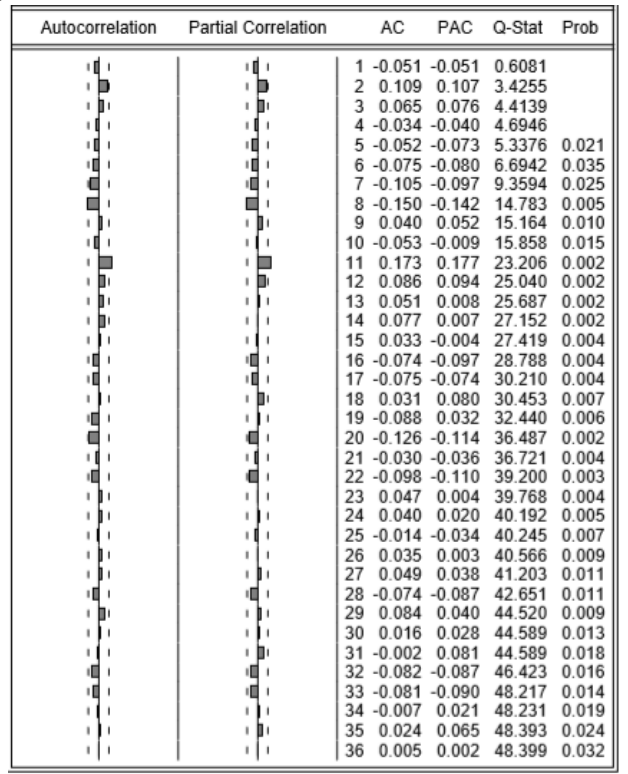

Fonte: Autores.

Todos os pressupostos sobre a utilização da metodologia adotada foram atendidos, sendo assim, tornou-se factível a realização da previsão da série in-sample (Figura 5), objetivando verificar a acurácia do modelo escolhido. Na Figura 5 a função de autocorrelação é representada pela sigla ACF e o \pm 2 S.E corresponde aos desvios padrões em relação a previsão da série in-sample.

Figura 5 - Previsão da série in-sample

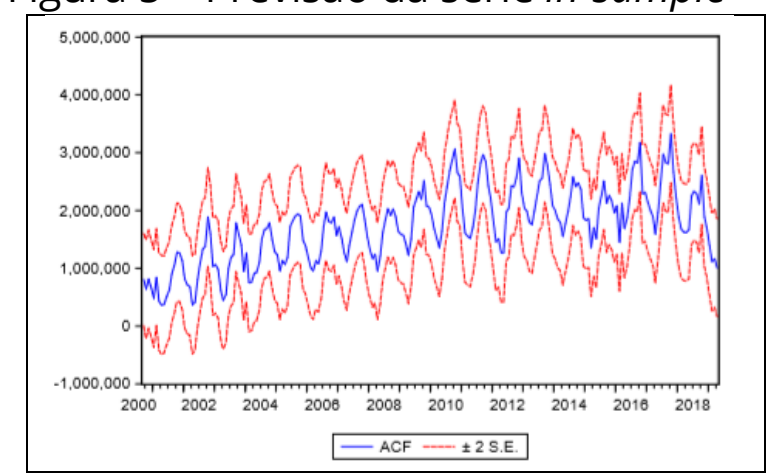

Fonte: Autores. 
Os valores da série original foram comparados com os valores estimados por meio do Mean Absolute Error (MAE), Mean Absolute Percentage Error (MAPE) e o coeficiente $U$-Theil (Tabela 4). A finalidade desta etapa é verificar a acurácia da previsão do modelo ajustado.

Tabela 4 - Medidas de Acurácia do Modelo Ajustado

\begin{tabular}{ll}
\hline SARIMA & $(1,1,1)(1,0,1)_{6}$ \\
\hline MAE & 292102,1 \\
MAPE & 20,1160 \\
U-THEIL & 0,09977 \\
\hline Fonte: Autores.
\end{tabular}

Pode-se dizer que o modelo SARIMA $(1,1,1)(1,0,1)_{6}$ apresenta resultados superiores em relação à previsão ingênua, uma vez que, o valor obtido pelo coeficiente U-Theil foi menor que 1 (0,09977). Com base nos resíduos do modelo, já que os mesmos são não autocorrelacionados, foi traçado o gráfico de controle de medidas individuais. Tal gráfico permitiu analisar cada uma das observações da série da exportação brasileira de açúcar compreendidas entre janeiro de 2000 a abril de 2019.

Figura 6 - Gráfico de controle de medidas individuais dos resíduos SARIMA $(1,1,1)$ $(1,0,1)_{6}$

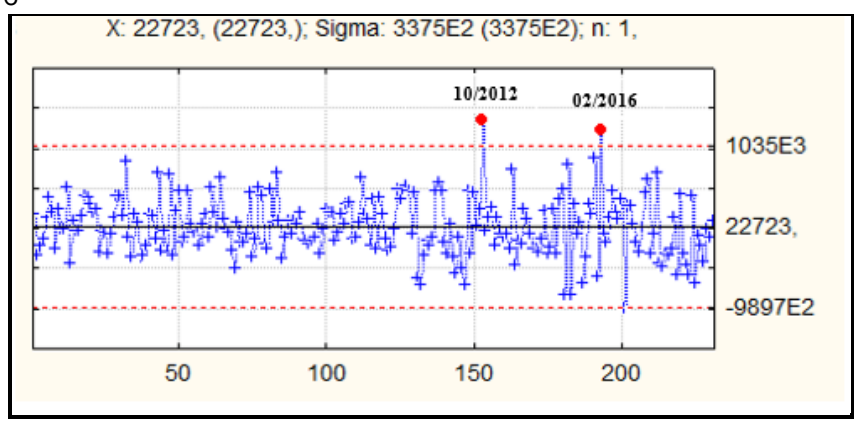

Na Figura 6, nota-se que os pontos estão oscilando em torno da média (linha central do gráfico), mostrando, assim, os períodos de alta e baixa na exportação do açúcar brasileiro. Os gráficos de controle apresentam dois limites - calculados a partir dos dados amostrais - que separam a variação aleatória da variação não-aleatória. O valor maior corresponde ao limite superior de controle (LSC) e o valor menor é chamado de limite inferior de controle (LIC), os mesmos estão localizados nas linhas pontilhas de vermelho no gráfico (REBELATO et al.,2006). Portanto, destacam-se apenas 2 outliers (pontos que estão em vermelho no gráfico), em outubro de 2012 e fevereiro 
de 2016, esses meses representam as observações 154 e 194, respectivamente, e estão fora dos limites superiores de controle, indicando que nesses pontos o processo está "fora de controle" ou a variação não ocorre de forma aleatória, e assim constata-se que nesses períodos a quantidade real de açúcar exportado foi muito maior que a quantidade prevista pelo modelo selecionado. Segundo Reuters (2016), isso deve-se ao preço do açúcar nesses períodos que fora muito atrativo para o produtor brasileiro e assim ocorreu um volume maior de exportações. Logo, compreender e prever o comportamento da exportação brasileira de açúcar é uma informação importante para a balança comercial do país, já que o açúcar é um dos principais produtos exportados no Brasil.

\section{CONSIDERAÇÕES FINAIS}

A metodologia de Box e Jenkins mostrou-se eficaz para compreender o comportamento da exportação brasileira de açúcar e, com isso, fornecer subsídios para tomada de decisões estratégicas no setor. Para analisar as oscilações da série, foram utilizados os modelos da classe geral SARIMA, pois a variável apresenta autocorrelação e efeitos de sazonalidade. Nesta pesquisa, a variável analisada foi a quantidade de açúcar exportada pelo país, descrita em valores mensais de janeiro de 2000 a abril de 2019.

O melhor modelo para prever a exportação brasileira de açúcar foi o SARIMA $(1,1,1)(1,0,1)_{6}$, selecionado com base nos valores dos critérios penalizadores AIC e BIC. Esse modelo foi utilizado para realizar a previsão, dado que a sua acurácia foi superior a previsão ingênua conforme o coeficiente $U$-Theil $=0,09977$. Ademais, as estatísticas de acurácia MAE e MAPE também confirmaram a performance de previsão do modelo selecionado. Ao analisar o gráfico de controle aplicado aos resíduos do modelo, verificou-se a presença de outliers no limite superior, em outubro de 2012 e fevereiro de 2016. Esses pontos caracterizaram uma exportação real do açúcar muito maior que exportação prevista. Assim, foi respondido a pergunta de pesquisa encontrada na parte introdutória do artigo, já que o monitoramento da exportação de açúcar no período em questão foi dado a partir do modelo SARIMA $(1,1,1)(1,0,1)_{6}$ e dos gráficos de controle gerados a partir dos resíduos deste modelo. 
Portanto, conhecer e prever o comportamento da exportação brasileira do açúcar é uma informação relevante para a balança comercial do país, dado que o açúcar é um dos principais produtos exportados. Sugere-se para estudos futuros realizar uma modelagem que englobe as variáveis influenciadoras da exportação do açúcar, como a produção de cana-de-açúcar, cotação do dólar e índices financeiros de países importadores. 
REFERÊNCIAS

AGRIC. Produção de Cana-de-açúcar. 2009. Disponível em: https://www.agric.com.br/producoes/cultivo_da_cana.html. Acesso em: 10/09/2019.

AKAIKE H. A new look at the statistical model identification. IEEE Transactions on Automatic Control. 1974; 19(6):716-723.

BOZDOGAN H. Model selection and Akaike's information criterion (AIC): The general theory and its analytical extensions. Psychometrica. 1987; (52):345-370.

BOX GEP, JENKINS GM. Time series analysis, forecasting and control. San Francisco: Holden Day, 1970.

BOX GEP, JENKINS GM, REINSEL GC. Time series analysis: forecasting and control. 3rd ed. New Jersey: Printice Hall, 1994.

DICKEY DA, FULLER WA. Likelihood ratio statistics for autoregressive time series with a unit root. Econometrica. 1981; 49(4):1057-1072.

FABER MEE. 0 ciclo do açúcar. 2016. Disponível em: http://www.historialivre.com/brasil/salaacucar.htm. Acesso em: 19/09/19.

FERLINI VLA. Açúcar e colonização.1 st ed. Alameda; 2010.

BORGES JM. Brasil deve perder liderança na produção de açúcar para Índia. 2018. Disponível em: https://g1.globo.com/economia/agronegocios/noticia/2018/09/05/brasil-deve-perderlideranca-na-producao-de-acucar-para-india.ghtml. Acesso em: 10/09/2019.

KWIATKOWSKI D, PHILLIPS CB, SCHMIDT P, SHIN Y. Testing the null hypothesis of stationarity against the alternative of a unit root. Journal of Econometrics. 1992; 54:159-178.

MONTGOMERY DC. Introduction to statistical quality control. 3rd ed. New York: John Wiley \& Sons, Inc. 1997.

MORETTIN PA. Econometria financeira: um curso em séries temporais financeira. 3rd ed. São Paulo: Blucher, 2016.

NOVACANA. A produção de cana-de-açúcar no Brasil (e no mundo). 2008. Disponível em: $\quad$ https://www.novacana.com/cana/producao-cana-de-acucar-brasil-e-mundo. Acesso em:10/09/2019. 
NOVA CANA. As projeções de produção de cana, açúcar e etanol para a safra 2023/24 da Fiesp/MB Agro. 2014. Disponível em: https://www.novacana.com/estudos/projecoes-producao-cana-acucar-etanol-safra2023-2024-fiesp-mb-agro. Acesso em: 10/09/2019.

REUTERS. Brasil deve perder liderança na produção de açúcar para Índia. 2018. Disponível em: https://g1.globo.com/economia/agronegocios/noticia/2018/09/05/brasil-deve-perderlideranca-na-producao-de-acucar-para-india.ghtml. Acesso em: 10/09/2019.

REUTERS. Brasil exporta em fevereiro maior volume de açúcar bruto e etanol desde 2013. 2016. Disponível em: https://br.reuters.com/article/domesticNews/idBRKCNOW35M7. Acesso em: 19/09/19.

SCHWARZ GE. Estimating the dimension of a model. Annals of Statistics. 1978;6(2):461-464.

SOUZA AM, LíRIO GW, KLIDZIO, R. Análise da estabilidade da produção de leite no município de Santo Ângelo - RS. Revista Ciência \& Natura 2012/13; 34(2):7-26.

SOUZA FM. Modelos de previsão: aplicações à energia elétrica ARIMA-ARCH-AI e ACP. 1 ed. Curitiba: Appris, 2016.

ÚNICA. Açúcar - Importante fonte de energia. 2019. Disponível em: https://www.unica.com.br/setor-sucroenergetico/acucar/. Acesso em: 10/09/2019. 